\title{
Effective Field Theory of ideal-fluid Hydrodynamics
}

\author{
Adriaan M. J. Schakel \\ Institut für Theoretische Physik \\ Freie Universität Berlin \\ Arnimallee 14, 14195 Berlin
}

(July 14, 1996)

\begin{abstract}
Starting from a standard description of an ideal, isentropic fluid, we derive the effective theory governing a gapless non-relativistic mode - the sound mode. The theory, which is dictated by the requirement of Galilei invariance, entails the entire set of hydrodynamic equations. The gaplessness of the sound mode is explained by identifying it as the Goldstone mode associated with the spontaneous breakdown of Galilei invariance. Differences with a superfluid are pointed out.
\end{abstract}

Typeset using REVTEX 
The hydrodynamics of an ideal, classical fluid was already well understood in the 19th century. The case of isentropic flow, for which the entropy per unit mass is constant, is particularly simple. The pressure $P$ is here a function of the mass density $\rho$ only, and the flow is automatically a potential flow. A property of such a fluid is that it supports unattenuated sound waves, i.e., propagating density oscillations. The waves are unattenuated because viscosity and thermal conductivity, which usually serve to dissipate the energy of a propagating mode, are absent. Sound waves belong to the class of modes having a gapless energy spectrum. The purpose of this essay is to argue that their presence in an isentropic fluid is an emergent property [1]. That is, we do not take the existence of this gapless mode for granted, but wish to explain it from an underlying principle, namely that of broken symmetry.

To describe the hydrodynamics of an isentropic fluid, we use Eckart's variational principle [2] and start with the Lagrange density

$$
\mathcal{L}=\frac{1}{2} \rho \mathbf{v}^{2}-\rho e+\theta\left[\partial_{0} \rho+\nabla \cdot(\rho \mathbf{v})\right]
$$

where $\mathbf{v}$ is the velocity field, $\rho$ the mass density, and $e$ the internal energy per unit mass. For isentropic flow $e$ is a function of $\rho$ alone. The first and second term in (国) represent the kinetic and potential energy density, respectively. The variable $\theta$ is a Lagrange multiplier introduced to impose the conservation of mass:

$$
\partial_{0} \rho+\nabla \cdot(\rho \mathbf{v})=0
$$

its dimension is $[\theta]=\mathrm{m}^{2} \mathrm{~s}^{-1}$. The variation of (1) with respect to $\mathbf{v}$ yields the equation

$$
\mathbf{v}=\nabla \theta
$$

It shows that, indeed, isentropic flow is automatically a potential flow and it also identifies the Lagrange multiplier $\theta$ as the velocity potential. (How to incorporate vortices in this variational approach will be discussed below.) With (3), the Lagrange density (1) becomes

$$
\mathcal{L}=-\rho\left[\partial_{0} \theta+\frac{1}{2}(\nabla \theta)^{2}+e\right]
$$


where we performed integrations by part. A second field equation can be obtained by varying the Lagrange density with respect to $\rho$. This yields the Bernoulli equation

$$
\partial_{0} \theta+\frac{1}{2}(\nabla \theta)^{2}+h=0
$$

with $h=\partial(\rho e) / \partial \rho$ the specific enthalpy. On taking the gradient of (5), and using that for isentropic flow one has the thermodynamic relation

$$
\nabla h=\frac{1}{\rho} \nabla P,
$$

with $P=\rho^{2} \partial e / \partial \rho$ the pressure, one obtains Euler's equation

$$
\partial_{0} \mathbf{v}+\frac{1}{2} \nabla \mathbf{v}^{2}+\frac{1}{\rho} \nabla P=0
$$

governing the flow of the fluid.

We next wish to investigate the symmetry content of the theory. Classical hydrodynamics has the following invariances:

- Invariance under space and time translations, $x_{\mu} \rightarrow x_{\mu}+\epsilon_{\mu}$, with $x_{\mu}=(t, \mathbf{x}), \mu=$ $0,1,2,3$ and $\epsilon_{\mu}$ a constant vector.

- Invariance under global translations of the velocity potential, $\theta \rightarrow \theta+\alpha$, with $\alpha$ a constant.

- Invariance under Galilei boosts,

$$
t \rightarrow t^{\prime}=t, \quad \mathbf{x} \rightarrow \mathbf{x}^{\prime}=\mathbf{x}-\mathbf{u} t ; \quad \partial_{0} \rightarrow \partial_{0}^{\prime}=\partial_{0}+\mathbf{u} \cdot \nabla, \quad \nabla \rightarrow \nabla^{\prime}=\nabla
$$

with $\mathbf{u}$ a constant velocity.

According to Noether's theorem, symmetries imply conservation laws. More specifically, the above invariances lead to the conservation laws:

- $\partial_{0} t_{0 j}+\partial_{i} t_{i j}=0$ and $\partial_{0} t_{00}+\partial_{i} t_{i 0}=0$, where $t_{\mu \nu}(\mu, \nu=0,1,2,3)$ is the energymomentum tensor, with $t_{0 i}=p_{i}$ the momentum density and $t_{00}=\mathcal{H}$ the Hamilton density; 
- $\partial_{0} j_{0}+\partial_{i} j_{i}=0$, with $j_{0}$ the mass density and $j_{i}$ the mass current;

- $\partial_{0} g_{0 j}+\partial_{i} g_{i j}=0$, with $g_{0 j}=-x_{j} j_{0}+t p_{j}$ and $g_{i j}=x_{i} j_{i}-t t_{i j}$ the corresponding charge densities and currents. Physically, the conservation $d G_{0 i} / d t=0$ of the charges $G_{0 i}=\int d^{3} x g_{0 i}$ means that the center of mass of the fluid, $\mathbf{X}=\int d^{3} x \mathbf{x} \rho / M$, with $M=\int d^{3} x \rho$ the total mass, moves with constant velocity,

$$
M \frac{d \mathbf{X}}{d t}=\int d^{3} x \mathbf{p} .
$$

Here, the right-hand side denotes the total momentum of the fluid.

From the Lagrange density (团) we obtain as explicit form for the various charge densities and currents [3]:

$$
\begin{aligned}
j_{0} & =-\frac{\partial \mathcal{L}}{\partial \partial_{0} \theta}=\rho \\
j_{i} & =-\frac{\partial \mathcal{L}}{\partial \partial_{i} \theta}=\rho v_{i} \\
t_{i j} & =\mathcal{L} \delta_{i j}-\frac{\partial \mathcal{L}}{\partial \partial_{i} \theta} \partial_{j} \theta=P \delta_{i j}+\rho v_{i} v_{j} \\
p_{j}\left(=t_{0 j}\right) & =-\frac{\partial \mathcal{L}}{\partial \partial_{0} \theta} \partial_{j} \theta=\rho v_{j} \\
t_{i 0} & =\frac{\partial \mathcal{L}}{\partial \partial_{i} \theta} \partial_{0} \theta=(\mathcal{H}+P) v_{i} \\
\mathcal{H}\left(=t_{00}\right) & =\frac{\partial \mathcal{L}}{\partial \partial_{0} \theta} \partial_{0} \theta-\mathcal{L}=\frac{\rho}{2} \mathbf{v}^{2}+\rho e .
\end{aligned}
$$

Time derivatives $\partial_{0} \theta$ have been eliminated through the field equation (5), so that, for example,

$$
\mathcal{L} \rightarrow \rho h-\rho e=\left(\rho \frac{\partial}{\partial \rho}-1\right)(\rho e)=P .
$$

A few remarks are in order. First, the Hamilton density $\mathcal{H}$ is the sum of the kinetic and potential energy density, as required. Second, the equivalence of the mass current $\mathbf{j}$ and the momentum density $\mathbf{p}$, which is a hallmark of Galilei invariance, is satisfied by the theory. Finally, the set of equations (10) constitutes all the equations of hydrodynamics. This brings 
us to the conclusion that the Lagrange density (4) encodes all the relevant equations for the description of an isentropic fluid.

We next turn to the description of sound waves. We will restrict ourselves to waves of small amplitude. These generate only small deviations in the density $\rho_{0}$ and pressure $P_{0}$ of the fluid at rest, so that we can expand the Lagrange density (田) in powers of $\rho-\rho_{0}=\tilde{\rho}$, with $|\tilde{\rho}|<<\rho_{0}$ :

$$
\mathcal{L}=-\left(\partial_{0} \theta+\frac{1}{2} \mathbf{v}^{2}\right)\left(\rho_{0}+\tilde{\rho}\right)-e_{0} \rho_{0}-h_{0} \tilde{\rho}-\frac{1}{2} h_{0}^{\prime} \tilde{\rho}^{2}+\mathcal{O}\left(\tilde{\rho}^{3}\right)
$$

The derivatives $\left({ }^{\prime}\right)$ with respect to $\rho$ are to be evaluated at $\rho=\rho_{0}$. Since for the system at rest $\theta$ is constant, it follows from (5) that $h_{0}=0$. If we denote the thermodynamic derivative $\partial P / \partial \rho$ by $c^{2}$, which has the dimension of a velocity squared, the coefficient of the quadratic term in $\tilde{\rho}$ can be written as

$$
h_{0}^{\prime}=\frac{1}{\rho_{0}} P_{0}^{\prime}=\frac{c_{0}^{2}}{\rho_{0}}
$$

Apart from an irrelevant constant term $\left(-e_{0} \rho_{0}\right)$ the Lagrange density becomes to this order

$$
\mathcal{L}=-\left(\partial_{0} \theta+\frac{1}{2} \mathbf{v}^{2}\right)\left(\rho_{0}+\tilde{\rho}\right)-\frac{c_{0}^{2}}{2 \rho_{0}} \tilde{\rho}^{2}
$$

We next eliminate $\tilde{\rho}$ by substituting

$$
\tilde{\rho}=-\frac{\rho_{0}}{c_{0}^{2}}\left(\partial_{0} \theta+\frac{1}{2} \mathbf{v}^{2}\right)
$$

which follows from expanding the field equation (5). Physically, this equation reflects Bernoulli's principle: in regions of rapid flow, the density $\rho=\rho_{0}+\tilde{\rho}$ and therefore the pressure is low. After eliminating $\tilde{\rho}$, we obtain a Lagrange density governing the velocity potential $\theta$ :

$$
\mathcal{L}_{\text {eff }}=-\rho_{0}\left(\partial_{0} \theta+\frac{1}{2} \mathbf{v}^{2}\right)+\frac{\rho_{0}}{2 c_{0}^{2}}\left(\partial_{0} \theta+\frac{1}{2} \mathbf{v}^{2}\right)^{2}
$$

This is the standard description of a gapless mode in a non-relativistic context [4,5]. The effective theory we derived here is apart from an irrelevant constant identical to a proposal by Takahashi [6] which was based on symmetry principles. 
The field equation one obtains for the velocity potential $\theta$ from (16) is non-linear:

$$
\rho_{0}\left(\partial_{0}^{2} \theta+\frac{1}{2} \partial_{0} \mathbf{v}^{2}\right)-\rho c_{0}^{2} \nabla \cdot \mathbf{v}+\frac{1}{2} \rho_{0}\left(\partial_{0} \mathbf{v}^{2}+\mathbf{v} \cdot \nabla \mathbf{v}^{2}\right)=0
$$

The information contained in this equation is nothing more than the conservation of mass because $\theta$ was initially introduced in (1) as a Lagrange multiplier precisely to enforce this conservation law. Indeed, remembering that the combination $\rho_{0}\left(\partial_{0} \theta+\frac{1}{2} \mathbf{v}^{2}\right)$ denotes $c_{0}^{2}$ times $\tilde{\rho}(t, \mathbf{x})=\rho(t, \mathbf{x})-\rho_{0}$, with $\rho_{0}$ the constant density of the fluid at rest, we see that (17) reproduces (21) in this approximation. To simplify (17), we replace $\rho_{0}$ in the first and last term by the full density $\rho$ (which is justified to this order) to arrive at the known [7], but unfamiliar field equation

$$
\partial_{0}^{2} \theta-c_{0}^{2} \nabla^{2} \theta=-\partial_{0} \mathbf{v}^{2}-\frac{1}{2} \mathbf{v} \cdot \nabla \mathbf{v}^{2}
$$

governing sound waves in the fluid. If we ignore the non-linear terms, it becomes the more familiar wave equation

$$
\partial_{0}^{2} \theta-c_{0}^{2} \nabla^{2} \theta=0
$$

implying a gapless linear spectrum, and identifying $c$, which was introduced via the thermodynamic derivative $\partial P / \partial \rho=c^{2}$, as the sound velocity.

The combination $\partial_{0} \theta+\frac{1}{2}(\nabla \theta)^{2}$ appearing in the description of a non-relativistic gapless field is dictated by Galilei invariance. To obtain the transformation property of the velocity potential $\theta$ under a Galilei boost (8) we note that since $\nabla \theta$ is a velocity field, $\nabla \theta(t, \mathbf{x}) \rightarrow$ $\nabla^{\prime} \theta^{\prime}\left(t^{\prime}, \mathbf{x}^{\prime}\right)=\nabla \theta(t, \mathbf{x})-\mathbf{u}$. This gives as transformation rule for $\theta$

$$
\theta(t, \mathbf{x}) \rightarrow \theta^{\prime}\left(t^{\prime}, \mathbf{x}^{\prime}\right)=\theta(t, \mathbf{x})-\mathbf{u} \cdot \mathbf{x}+f(t)
$$

with $f(t)$ a yet undetermined function of time. To determine $f(t)$ we note that the factor $-\partial_{0} \theta$ in the Lagrange density (4) is the chemical potential per unit mass. Indeed, using the standard definition $\mu=\partial \mathcal{H} / \partial \rho$, we find

$$
\mu=\frac{1}{2} \mathbf{v}^{2}+h=-\partial_{0} \theta
$$


where in the second equality we used the field equation (5). This identification fixes the transformation rule of $-\partial_{0} \theta$ :

$$
-\partial_{0} \theta(t, \mathbf{x}) \rightarrow-\partial_{0}^{\prime} \theta^{\prime}\left(t^{\prime}, \mathbf{x}^{\prime}\right)=-\partial_{0} \theta(t, \mathbf{x})-\mathbf{u} \cdot \mathbf{v}(t, \mathbf{x})+\frac{1}{2} \mathbf{u}^{2}
$$

and in combination with (20), yields for $f(t)$

$$
\partial_{0} f(t)=\frac{1}{2} \mathbf{u}^{2}, \quad \text { or } \quad f(t)=\frac{1}{2} \mathbf{u}^{2} t
$$

up to an irrelevant constant. It is easily checked that both the combination $\partial_{0} \theta+\frac{1}{2}(\nabla \theta)^{2}$ appearing in the effective theory (16) as well as the field equation (18) are invariant under Galilei boosts. So, contrary to what is sometimes stated in the literature [8], sound waves are invariant under Galilei boosts. The wave equation (19) is, of course, not invariant because essential non-linear terms are omitted.

From the effective theory (16) one can again calculate the various Noether charge densities and currents [6]. They are, as might be expected, of the same form as the exact expressions (10), but now with the approximations

$$
\rho \simeq \rho_{0}-\frac{\rho_{0}}{c_{0}^{2}}\left(\partial_{0} \theta+\frac{1}{2} \mathbf{v}^{2}\right)
$$

as follows from Eq. (15),

$$
\mathcal{H} \simeq \frac{\rho}{2} \mathbf{v}^{2}+\frac{c_{0}^{2}}{2 \rho_{0}}\left(\rho-\rho_{0}\right)^{2}
$$

and

$$
P \simeq-\rho_{0}\left(\partial_{0} \theta+\frac{1}{2} \mathbf{v}^{2}\right) \simeq c_{0}^{2}\left(\rho-\rho_{0}\right)
$$

This last equation is consistent with the expression one obtains from directly expanding the pressure: $P(\rho)=P_{0}+\tilde{\rho} P_{0}^{\prime}$ since $P_{0}=0$ and $P_{0}^{\prime}=c_{0}^{2}$.

To recapitulate, we have derived an effective theory describing a massless mode starting from the Lagrange density (4) which entails the complete hydrodynamics of an isentropic fluid. We now wish to argue that this massless mode is a Goldstone mode associated 
with a spontaneously broken symmetry. A first indication in favor of such an interpretation follows because the Hamilton density $\mathcal{H}$ displays a property typical for a system with broken symmetry, namely that it is not a function of the velocity potential itself, but is a function of the gradient of the field. The energy is minimal if $\theta$ is uniform throughout the sample, i.e., there is rigidity [1]. A second indication comes from the observation that precisely the same effective theory as (16) arises in the context of (fermionic and bosonic) superfluids [5], and there $\theta$ has been identified as a Goldstone mode.

Usually the broken symmetry can be identified by the general property that the Goldstone mode is translated under the broken symmetry operations. (There may be other effects too, but the translation is always there.) Here, this general characteristic does not uniquely identify the broken symmetry because $\theta$ is translated under two symmetry operations. According to the transformation rule $(20)$ with $f(t)$ given in $(23)$, we have that under a Galilei boost

$$
\delta_{\mathbf{u}}^{G} \theta(t, \mathbf{x}):=\theta^{\prime}(t, \mathbf{x})-\theta(t, \mathbf{x})=-\mathbf{u} \cdot \mathbf{x}+t \mathbf{u} \cdot \nabla \theta(t, \mathbf{x})
$$

where we took the transformation parameter $\mathbf{u}$ infinitesimal small so that quadratic and higher powers in $\mathbf{u}$ may be ignored. The first term at the right-hand side shows that the velocity potential is translated under a Galilei boost. The second symmetry under which $\theta$ is translated is generated by the total mass $M=\int d^{3} x \rho$. To see this we first compute from (目) the conjugate momentum $\pi_{\theta}$ of $\theta$

$$
\pi_{\theta}=\frac{\delta \mathcal{L}_{\text {eff }}}{\delta \partial_{0} \theta}=-\rho
$$

and note that $\theta$ and $\rho$ are canonically conjugate [9]:

$$
\left\{\theta(t, \mathbf{x}), \rho\left(t, \mathbf{x}^{\prime}\right)\right\}=-\delta\left(\mathbf{x}-\mathbf{x}^{\prime}\right)
$$

where $\{$,$\} denotes the Poisson bracket. We then use a central result of classical field theory$ stating that the charge $Q$ of a continuous symmetry is the generator of the corresponding transformations of the fields, $\chi(t, \mathbf{x}) \rightarrow \chi^{\prime}(t, \mathbf{x})$. More specifically, for an infinitesimal transformation $\delta_{\alpha}^{Q} \chi(t, \mathbf{x})=\chi^{\prime}(t, \mathbf{x})-\chi(t, \mathbf{x})$ one has 


$$
\delta_{\alpha}^{Q} \chi(t, \mathbf{x})=-\alpha\{\chi(t, \mathbf{x}), Q\}
$$

with $\alpha$ the transformation parameter. Equation (29) now immediately shows that under the symmetry generated by the total mass, $\theta$ is indeed translated

$$
\delta_{\alpha}^{M} \theta(t, \mathbf{x})=-\alpha\{\theta(t, \mathbf{x}), M\}=\alpha .
$$

The point is that the generators of Galilei boosts $G_{0 j}=\int d^{3} x\left(-x_{j} j_{0}+t p_{j}\right)$ also contain the mass density $\rho=j_{0}$. It is therefore impossible to distinguish a broken Galilei invariance from a broken mass symmetry by considering the algebra alone.

Let us at this point pause and consider the case of a superfluid. It is well established that in the normal-to-superfluid phase transition, the mass symmetry is spontaneously broken. It is helpful to recall that this comes about because in a superfluid, which is a quantum system, many particles Bose-Einstein condense in a single quantum state. This coherence allows us to describe the system by a complex field $\psi(t, \mathbf{x})=\sqrt{\rho(t, \mathbf{x}) / m} \exp [i m \varphi(t, \mathbf{x}) / \hbar]$ normalized such that $\psi^{*} \psi$ yields the particle number density $\rho / m$ of the condensate. The field $\varphi$, which will turn out to describe the Goldstone mode of the broken mass symmetry, is a phase field and therefore compact. The field $\psi$ is usually referred to as the condensate wavefunction, even in the modern literature [10,11]. In our view, this is somewhat misleading. As has been stressed by Feynman [12], $\psi$ is a classical field describing the coherent behavior of many condensed particles in the same way as the classical gauge potential of electrodynamics describes the behavior of many photons in a single state. For these classical fields there is no probability interpretation as is required for wavefunctions [13].

It has been argued by Feynman [12] that the $\psi$-field is governed by a non-relativistic $\left(\psi^{*} \psi\right)^{2}$-theory defined by the Lagrange density

$$
\mathcal{L}_{\psi}=i \hbar \psi^{*} \partial_{0} \psi-\frac{\hbar^{2}}{2 m}|\nabla \psi|^{2}-\frac{c_{0}^{2}}{2 \rho_{0}}\left(m \psi^{*} \psi-\rho_{0}\right)^{2} .
$$

The potential energy has its minimum along a circle away from the origin at $\psi^{*} \psi=\rho_{0}$. This implies a spontaneous breakdown of the mass symmetry. The Lagrange density (32) reduces 
to the one given in (14) when the term $-\hbar^{2}(\nabla \rho)^{2} / 8 m^{2} \rho$ is ignored. Using the expression for the pressure, cf. (11)

$$
P=\left[\rho\left(\frac{\partial}{\partial \rho}-\partial_{i} \frac{\partial}{\partial \partial_{i} \rho}\right)-1\right](\rho e),
$$

we find that this term gives the contribution $-\hbar^{2}\left(\nabla^{2} \rho\right) / 4 m^{2}$ to the pressure - the so-called quantum pressure. The reason for calling it this way is that it is the only place where Planck's constant appears in the equations. To the order in which we are working, it is consistent to ignore this term. Because the field equation for $\psi$ derived from (32) has the form of a non-linear Schrödinger equation, we will refer to $\psi$ as a Schrödinger field. We trust however that the reader realizes that it is a classical field unrelated to a Schrödinger wavefunction.

Let us compare the transformation properties of the Schrödinger field with that of the velocity potential $\theta$ of an isentropic fluid. Under a Galilei boost, $\psi(t, \mathbf{x})$ transforms as 13

$$
\psi(t, \mathbf{x}) \rightarrow \psi^{\prime}\left(t^{\prime}, \mathbf{x}^{\prime}\right)=\exp \left[i\left(-\mathbf{u} \cdot \mathbf{x}+\frac{1}{2} \mathbf{u}^{2} t\right) m / \hbar\right] \psi(t, \mathbf{x})
$$

With $m \varphi / \hbar$ denoting the phase of the Schrödinger field, we see that $\varphi$ transforms in the same way as does the velocity potential.

Using that the canonical conjugate of the $\psi$-field is $\pi_{\psi}=i \hbar \psi^{*}$, we easily derive the Poisson bracket

$$
\{\psi(t, \mathbf{x}), M\}=-i(m / \hbar) \psi(t, \mathbf{x})
$$

This shows that the total mass $M$ generates phase transformations on the $\psi$-field: $\psi(t, \mathbf{x}) \rightarrow$ $\psi^{\prime}(t, \mathbf{x})=\exp (i \alpha m / \hbar) \psi(t, \mathbf{x})$. The phase $\varphi$ of the Schrödinger field is consequently translated under the symmetry, just like the velocity potential $\theta$. This transformation property identifies $\varphi$ as the Goldstone mode of the broken mass symmetry. The Poisson bracket (35) also implies that $\varphi$ and $\rho$ are canonical conjugate [1], cf. (29)

$$
\left\{\varphi(t, \mathbf{x}), \rho\left(t, \mathbf{x}^{\prime}\right)\right\}=-\delta\left(\mathbf{x}-\mathbf{x}^{\prime}\right)
$$


A similar relation holds for superconductors. On quantizing, the Poisson bracket is replaced by a commutator. The Heisenberg uncertainty relation that results for the conjugate pair has recently been demonstrated experimentally [14].

As we remarked above, a necessary condition for the breaking of the mass symmetry is the presence of a condensate. Such an intrinsic quantum phenomenon, requiring many particles in a single state, has no analog in a classical setting. Hence, the mass symmetry cannot be broken in classical hydrodynamics. This leaves us with the second possibility, namely that of a spontaneously broken Galilei invariance. The breakdown is a result of the presence of a finite mass density. This can be inferred [6] from considering the transformation of the momentum density $\mathbf{p}(x)$ under a Galilei boost:

$$
\delta_{\mathbf{u}}^{G} p_{i}(t, \mathbf{x})=-u_{j}\left\{p_{i}(t, \mathbf{x}), G_{0 j}(t)\right\}=-u_{i} \rho(t, \mathbf{x})+t \mathbf{u} \cdot \nabla p_{i}(t, \mathbf{x})
$$

or with $\delta_{\mathbf{u}}^{G}=u_{j} \delta_{j}^{G}$

$$
\delta_{j}^{G} p_{i}(t, \mathbf{x})=-\rho(t, \mathbf{x}) \delta_{j i}+t \partial_{j} p_{i}(t, \mathbf{x})
$$

If the mass density $\rho$ is finite, the left-hand side is non-zero, which is a symmetry-breaking condition.

There is an essential difference between the breaking of Galilei invariance and that of mass symmetry. Although both symmetries are Abelian, the mass symmetry is a compact $\mathrm{U}(1)$ symmetry, whereas the Galilei group is non-compact. More specifically, the transformation parameter $\alpha$ of the $\mathrm{U}(1)$ group has a finite domain $(0 \leq \alpha<2 \pi)$, while the domain of $\mathbf{u}$, the transformation parameter of the Galilei group, is infinite. This is closely connected with the impossibility to represent the velocity potential of classical hydrodynamics as the phase of a complex field. An immediate physical manifestation of the difference is that a system with broken U(1) invariance supports topologically stable vortices, whereas a system with broken Galilei invariance does not. This is not to say that vortices are absent in the latter case, it merely states that their stability is not guaranteed by topological conservation laws. Closely connected to this is that the circulation is not quantized in classical hydrodynamics, which is 
known to exist in superfluids. Yet, the circulation is conserved also in isentropic fluids. This is again not for topological, but for dynamical reasons. More specifically, the conservation is proven by invoking Euler's equation (17) as was first done for an ideal, incompressible fluid by Helmholtz [15] and generalized to a compressible fluid by Thomson [16].

The easiest way to observe vortices in a classical fluid is to punch a hole in the bottom of the vessel containing the fluid. As the fluid pours out a vortex is formed in the remaining fluid - a phenomenon daily observed by people unplugging a sinkhole. Often, as happens in, for example, superfluid ${ }^{4} \mathrm{He}$, the core of a vortex is in the normal state so that the symmetry is restored there. In the present context this would mean that inside the vortex core, the fluid mass density $\rho$ is zero. This is indeed what is observed: the vortex core consists of air, therefore no fluid is present and $\rho=0$ there.

In the eye of a tropical cyclone - another example of a vortex, nature does its best to restore the Galilei symmetry, record low atmospheric pressures being measured there. (A complete restoration would imply the absence of air corresponding to zero pressure.)

It is customary to incorporate vortices in a potential flow via the introduction of socalled Clebsch potentials [17]. We will not follow this route, but instead use the principle of vortex gauge symmetry [18]. In this approach, one introduces a so-called vortex gauge field $\theta_{\mu}^{\mathrm{P}}=\left(\theta_{0}^{\mathrm{P}}, \boldsymbol{\theta}^{\mathrm{P}}\right)$ in the Lagrange density via minimally coupling to the Goldstone field:

$$
\partial_{\mu} \theta \rightarrow \partial_{\mu} \theta+\theta_{\mu}^{\mathrm{P}}
$$

with $\partial_{\mu}=\left(\partial_{0},-\nabla\right)$ and

$$
\nabla \times \boldsymbol{\theta}^{\mathrm{P}}=-2 \boldsymbol{\omega}
$$

so that $\nabla \times \mathbf{v}=2 \boldsymbol{\omega}$ yields (twice) the vorticity $\boldsymbol{\omega}$ of the vortex. The object $\theta_{\mu}^{\mathrm{P}}$ describing the vortex is called vortex gauge field $\left[18\right.$. The combination $\partial_{\mu} \theta+\theta_{\mu}^{\mathrm{P}}$ is invariant under the local gauge transformation

$$
\theta(t, \mathbf{x}) \rightarrow \theta(t, \mathbf{x})+\alpha(t, \mathbf{x}) ; \quad \theta_{\mu}^{\mathrm{P}} \rightarrow \theta_{\mu}^{\mathrm{P}}-\partial_{\mu} \alpha(t, \mathbf{x})
$$


with $\theta_{\mu}^{\mathrm{P}}$ playing the role of a gauge field. The left-hand side of (40) may be thought of as defining the "magnetic field" associated with the vortex gauge field $\mathbf{B}^{\mathrm{P}}=\nabla \times \boldsymbol{\theta}^{\mathrm{P}}$.

For illustrative purposes, let us consider an external, static vortex with circulation $\Gamma$ located along a line $L$, which may be closed or infinitely long [15]. Then, $\boldsymbol{\omega}=\frac{1}{2} \Gamma \boldsymbol{\delta}(L)$, where $\boldsymbol{\delta}(L)$ is a delta function on the line $L$,

$$
\delta_{i}(L)=\int_{L} d y_{i} \delta(\mathbf{x}-\mathbf{y})
$$

This model with a static, external vortex may be thought of as describing the steady flow in the presence of a vortex pinned to a fixed impurity. The field equation for $\theta$ obtained after the substitution (39) reads

$$
\partial_{0} \mu+c_{0}^{2} \nabla \cdot \mathbf{v}=\partial_{0} \mathbf{v}^{2}+\frac{1}{2} \mathbf{v} \cdot \nabla \mathbf{v}^{2}-\mathbf{v} \cdot \mathbf{E}^{\mathrm{P}}
$$

with $\mu=-\left(\partial_{0} \theta+\theta_{0}^{\mathrm{P}}\right)$ the chemical potential and $\mathbf{v}=\nabla \theta-\boldsymbol{\theta}^{\mathrm{P}}$ the velocity of the flow in the presence of the vortex. The last term gives a coupling of the velocity field to the "electric field" associated with $\theta_{\mu}^{\mathrm{P}}$,

$$
\mathbf{E}^{\mathrm{P}}=-\nabla \theta_{0}^{\mathrm{P}}-\partial_{0} \boldsymbol{\theta}^{\mathrm{P}}
$$

We note that the field equation (43) is invariant under local vortex gauge transformations. Ignoring the higher-order terms and choosing the gauge $\theta_{0}^{\mathrm{P}}=0$, we obtain as equation for the flow in the presence of a static vortex:

$$
\nabla \cdot \mathbf{v}=0, \quad \text { or } \quad \nabla \cdot\left(\nabla \theta-\boldsymbol{\theta}^{\mathrm{P}}\right)=0
$$

which is solved by

$$
\theta(\mathbf{x})=-\int d^{3} y G(\mathbf{x}-\mathbf{y}) \nabla \cdot \boldsymbol{\theta}^{\mathrm{P}}(\mathbf{y})
$$

Here, $G(\mathbf{x})$ is the Green function of the Laplace operator

$$
G(\mathbf{x})=\int \frac{d^{3} k}{(2 \pi)^{3}} \frac{\mathrm{e}^{i \mathbf{k} \cdot \mathbf{x}}}{\mathbf{k}^{2}}=\frac{1}{4 \pi|\mathbf{x}|}
$$


Straightforward manipulations then yield the well-known Biot-Savart law for the velocity field in the presence of a static vortex 15, 18

$$
\mathbf{v}_{\mathbf{v}}(\mathbf{x})=\frac{\Gamma}{4 \pi} \int_{L} d \mathbf{y} \times \frac{\mathbf{x}-\mathbf{y}}{|\mathbf{x}-\mathbf{y}|^{3}}
$$

where the integration is along the vortex. This exemplifies the viability of the vortex gauge principle as an alternative to describe vortices in a potential flow.

Let us continue to study the dynamics of vortices - a subject that has recently received considerable attention in the literature [19]. Because the vortex motion is determined by the flow itself, the vortex can no longer be considered as external. We shall see that the non-linear part of the field equation (43) becomes relevant here.

In the absence of external forces, the vortex moves with a constant velocity, $\mathbf{v}_{L}$ say. The flow in the presence of a moving vortex can be obtained from the static solution (48) by replacing the coordinate $\mathbf{x}$ with $\mathbf{x}-\mathbf{v}_{L} t$. This implies that

$$
\partial_{0} \mathbf{v}_{\mathrm{v}}=-\mathbf{v}_{L} \cdot \nabla \mathbf{v}_{\mathrm{v}}=-\nabla\left(\mathbf{v}_{L} \cdot \mathbf{v}_{\mathrm{v}}\right)
$$

To study sound waves in the presence of a moving vortex, we write the velocity field as $\mathbf{v}(t, \mathbf{x})=\mathbf{v}_{\mathbf{v}}\left(\mathbf{x}-\mathbf{v}_{L} t\right)+\nabla \tilde{\theta}(t, \mathbf{x})$, with $\tilde{\theta}$ describing small variations around the moving vortex solution. Equation (49) then requires that we write for the chemical potential in (43)

$$
\mu(t, \mathbf{x})=\mathbf{v}_{L} \cdot \mathbf{v}_{\mathbf{v}}\left(\mathbf{x}-\mathbf{v}_{L} t\right)-\partial_{0} \tilde{\theta}(t, \mathbf{x})
$$

This leads to the linearized field equation in the frame moving with the vortex [19]

$$
\partial_{0}^{2} \tilde{\theta}(t, \mathbf{x})-c_{0}^{2} \nabla^{2} \tilde{\theta}(t, \mathbf{x})=-\mathbf{v}_{\mathbf{v}}(\mathbf{x}) \cdot \nabla \partial_{0}[2 \tilde{\theta}(t, \mathbf{x})-\tilde{\theta}(t, 0)]
$$

describing sound waves in the presence of a moving vortex. We have suppressed the prime on the coordinate $\mathbf{x}^{\prime}=\mathbf{x}-\mathbf{v}_{L} t$ of the moving frame. In deriving (51) we again used the gauge $\theta_{0}^{\mathrm{P}}=0$, and replaced in the last term $\mathbf{v}_{L}$ with $\nabla \tilde{\theta}(t, 0)$. This is justified because the vortex is carried by the sound wave and in the moving frame, $\mathbf{x}=0$ denotes the location of the vortex. The first term at the right-hand side in (51) stems from the non-linear term $\partial_{0} \mathbf{v}^{2}$ in 
the general field equation (43). Thus the non-linearity of sound waves becomes detectable. Equation (51) can be used as a basis to study the scattering of phonons of a free moving vortex [19].

So far we have contrasted the breakdown of Galilei invariance (caused by a finite density) and mass symmetry (caused by a non-zero condensate). A superfluid, however, has a finite density as well as a non-zero condensate. Both symmetries are therefore broken and we expect two different Goldstone modes to be present. This is indeed what is observed in superfluid ${ }^{4}$ He. The system supports besides first sound, which are the usual density waves associated with the breakdown of Galilei invariance, also second sound, or entropy waves. The latter mode depends crucially on the presence of the condensate and is the Goldstone mode associated with the breakdown of mass symmetry. At the transition point, the second sound velocity vanishes, whereas the first sound velocity remains finite. This is as expected since only the condensate vanishes at the superfluid phase transition; the total mass density remains finite.

\section{ACKNOWLEDGMENTS}

It is a pleasure to thank H. Kleinert and G. Vasconcelos for useful discussions. 


\section{REFERENCES}

[1] P. W. Anderson, Basic Notions of Condensed Matter Physics (Benjamin-Cummings, Menlo Park, Ca, 1984).

[2] C. Eckart, Phys. Rev. 54 (1938) 920; W. Yourgrau and S. Mandelstam, Variational Principles in Dynamics and Quantum Theory (Pitman, London, 1968).

[3] R. Kronig and A. Thellung, Physica 18 (1952) 749.

[4] M. Greiter, F. Wilczek, and E. Witten, Mod. Phys. Lett. B 3 (1989) 903.

[5] A. M. J. Schakel, Mod. Phys. Lett. B 4 (1990) 927; 5 (1991) 833; Proceedings of the Körber Symposium on Superfluid ${ }^{3}$ He in Rotation, Helsinki, 1991, edited by M. M. Salomaa, Physica B 178 (1992) 280; Int. J. Mod. Phys. B 8 (1994) 2021.

[6] Y. Takahashi, Fortschr. Phys. 36 (1988) 63.

[7] See, for example, B. K. Shivamoggi, Theoretical Fluid Dynamics (Martinus Nijhoff, Dordrecht, 1985), p. 191, and references therein.

[8] See, for example, J. D. Jackson, Classical Electrodynamics (Wiley, New York, 1975), p. 505.

[9] F. London, Superfluids (Wiley, New York, 1954), vol. II.

[10] I. J. Aitchison, P. Ao, D. J. Thouless, and X. -M. Zhu, Phys. Rev. B 51 (1995) 6531.

[11] M. Stone, Dynamics of $T=0$ BCS Condensates, eprint: cond-mat/9409124.

[12] R. P. Feynman, Statistical Mechanics (Addison Weseley, New York, 1972) Sec. 10.12.

[13] E. Fick, Einführing in die Grundlagen der Quantentheorie (Akademische Verlagsgesellschaft, Frankfurt am Main, 1968).

[14] W. J. Ellon, M. Matters, U. Geigenmüller, and J. E. Mooij, Nature 371 (1994) 594.

[15] H. von Helmholtz, Journal für die reine und angewandte Mathematik 55 (1858) 25. 
[16] W. Thomson, Trans. Roy. Soc. Edingb. 25 (1869) 217.

[17] A. Clebsch, Journal für die reine und angewandte Mathematik 56 (1859) 1; H. Lamb, Hydrodynamics (Cambridge University Press, Cambridge, 1942) p. 248.

[18] H. Kleinert, Int. J. Mod. Phys. A 7, 4693 (1992); Gauge Fields in Condensed Matter (World Scientific, Singapore, 1989).

[19] E. B. Sonin, The Magnus force in superfluids and superconductors, eprint: condmat/9606099, and references therein. 